\title{
Entropy, holography and the second law
}

\author{
Daniel R. Terno \\ Perimeter Institute for Theoretical Physics, 35 King St. N., Waterloo, Ontario, Canada N2J 2 W9
}

\begin{abstract}
The geometric entropy in quantum field theory is not a Lorentz scalar and has no invariant meaning, while the black hole entropy is invariant. Renormalization of entropy and energy for reduced density matrices may lead to the negative free energy even if no boundary conditions are imposed. Presence of particles outside the horizon of a uniformly accelerated observer prevents the description in terms of a single Unruh temperature.

PACS numbers: 04.70.Dy, 03.67.-a, 04.60.-m
\end{abstract}

Entropy plays a central role in statistical physics, thermodynamics and information theory [1, 2, 3, 4, 5. The von Neumann entropy of a quantum system that is described by a density operator $\rho$,

$$
S=-\operatorname{tr} \rho \log \rho,
$$

has a simple information-theoretical meaning. Each measurement scheme leads to a probability distribution $\left\{p_{\mu}\right\}$ on its outcomes, and its Shannon's entropy is

$$
H=-\sum_{\mu} p_{\mu} \log p_{\mu}
$$

The von Neumann entropy of the state $\rho$ is the lowest value that can be reached by the Shannon entropy over all possible probability distributions. The concepts of quantum information theory, with suitable adjustments, can be applied to any quantum system, including field theories on a curved background $[5,6]$.

To connect von Neumann's entropy with that of thermodynamics, a number of assumptions should be made [1, 2, 4, 7]. They are natural for a normal matter, and there are no experimental results that may hint at their possible violation. However, rigorous proofs exist only for a small number of simple models [2, 7].

Black holes are usually considered thermodynamical systems in their own right [8, 9]. Their entropy is one quarter of their horizon area in Plank units,

$$
S_{\mathrm{BH}}=A^{2} / l_{P}^{2},
$$

and their temperature is proportional to the surface gravity [10, 11]. This connection between area and entropy is elevated to a general physical law in different versions of the holographic principle [9, 12] that bounds the entropy inside a volume by the quarter of its area (the definitions of entropy and area vary, however). Perhaps the most striking consequence of the proportionality of entropy and area is the derivation [13] of the Einstein equations from the holographic principle together with the thermodynamic relation $\delta Q=T d S$.

The notion of reduced density operator and its entropy is fundamental for entanglement theory and quantum information in general [4, [5]. It is obtained by tracing out the irrelevant degrees of freedom (in the Hilbert space language) 4] or by restricting states to the local algebra of observables (in the framework of algebraic quantum theory) 14]. For pure states the von Neumann entropy of a reduced density operator is the measure of entanglement between the degrees of freedom "here" and "elsewhere". This is what is meant by the geometric entropy [15, 16]. The actual calculations of reduced density operators are quite complicated. Only the analysis for a half-space in the Minkowski spacetime (where $\rho$ can be obtained from the Rindler quantization and is related to the Unruh effect $[8,17]$ ) is relatively straightforward. The geometric entropy usually diverges. However, it was shown in a number of cases [15, 19, 20] that the regularized geometric entropy is proportional to the boundary area.

In this Letter we discuss some properties of entropy and its relation to thermodynamics. We show that the geometric entropy is not a Lorentz scalar for essentially the same reasons as the spin entropy of massive particles [5]. We consider the implications of the presence of Minkowski particles beyond the horizon of an accelerated observer. Her temperature is defined [1] by

$$
\frac{1}{T}=\frac{\partial S}{\partial E},
$$

using the renormalized energy and entropy. This temperature is different from the Unruh temperature for the same proper acceleration. We furthermore connect these results to the generalized second law, the derivation of the Einstein equations from thermodynamics and the microscopic origins of the black hole entropy.

Let us assume that splitting into "inside" and "outside" degrees of freedom allows writing a Fock space of some free quantum field theory as $\mathcal{F}(\mathcal{H}) \simeq \mathcal{F}_{1}\left(\mathcal{H}_{1}\right) \otimes$ $\mathcal{F}_{2}\left(\mathcal{H}_{2}\right)$. This decomposition may be associated either with spatial regions on a given time slice or with spacetime domains. The Lorentz-invariant vacuum state

$$
|\Omega\rangle=\sum_{i j} c_{i j}\left|\psi_{i}^{1}\right\rangle \otimes\left|\psi_{j}^{2}\right\rangle,
$$

has an explicit entangled form [5, 18, 19], and this is also true for one- and many-particle states. The reduced 
density matrix that corresponds to tracing out the "outside" degrees of freedom (that are labelled by "2") is $\rho_{i j}^{1}=\sum_{k} c_{i k} c_{j k}^{*}$.

Proposition. The reduced density matrix $\rho_{i j}$ is not covariant under Lorentz transformations.

For $\rho$ to have a definite transformation law, it is necessary for a unitary transformation on the entire space, $U(\Lambda)$, to be a direct product of unitaries that act on the "inside" and "outside" spaces, $U(\Lambda)=U_{1}(\Lambda) \otimes U_{2}(\Lambda)$. The vacuum state spans a one-dimensional irreducible representation space of the Poincare group. The direct product structure is compatible with this property of $|\Omega\rangle$ only if both representations $U_{1}$ and $U_{2}$ are onedimensional. However, the ensuing product structure of the vacuum is incompatible with the long-range correlations in it, and, in particular, with the violation of the Bell-type inequalities [5, 21]. A similar argument applies to one-particle states, which are faithful irreducible representations of the Poincare group and, therefore, cannot be described as a direct product of the two representations.

Hence, unlike the full state that transforms unitarily, its reduced density matrices do not have a definite Lorentz transformation law, and the entropy is not necessarily invariant. A byproduct of the non-invariance of entropy is that the (effective) number of degrees of freedom, $N=e^{S_{\max }}$, is frame-dependent.

Consider a massless scalar field in a box of the size $L$ in Minkowski spacetime. Compliance with the spacelike holographic bound [20] is enforced by imposing two cut-offs. First, one must limit the maximal frequency of each mode at about the Planck frequency $1 / l_{P}$. Then, in order to ensure gravitational stability only the states below a certain maximal energy are allowed. This bound is expressed (in the suitable units) as a dimensionless ratio $B \sim L / l_{P}$. As a result, in the rest frame of the box the maximal entropy is finite and proportional to the surface area, $S_{\max }=\ln N \propto L^{2} / l_{P}^{2}, 20$.

Now consider another observer that moves along one of the box's edges with some velocity $v>0$. In his frame the surface area is $A^{\prime}=2 L^{2}(1+2 / \gamma)$, where $\gamma=1 / \sqrt{1-v^{2}}$. The number of admissible modes should be lowered by about a factor of $\gamma(1+v)$ because of the Doppler effect. Hence, following the derivations of Ref. [20] the new maximal entropy is

$$
S_{\max }^{\prime}=S_{\max } / \sqrt{\gamma(1+v)} .
$$

In this example the maximal entropy is reduced faster than the surface area, so the spacelike holographic bound is satisfied for all velocities $v$.

An isolated stationary black hole furnishes an example of the invariant entropy. Consider two observers at the same spacetime point outside the horizon, but not necessarily in the asymptotically flat region. Their local coordinate frames are connected by some Lorentz transformation $\Lambda$. The global role of this local Lorentz

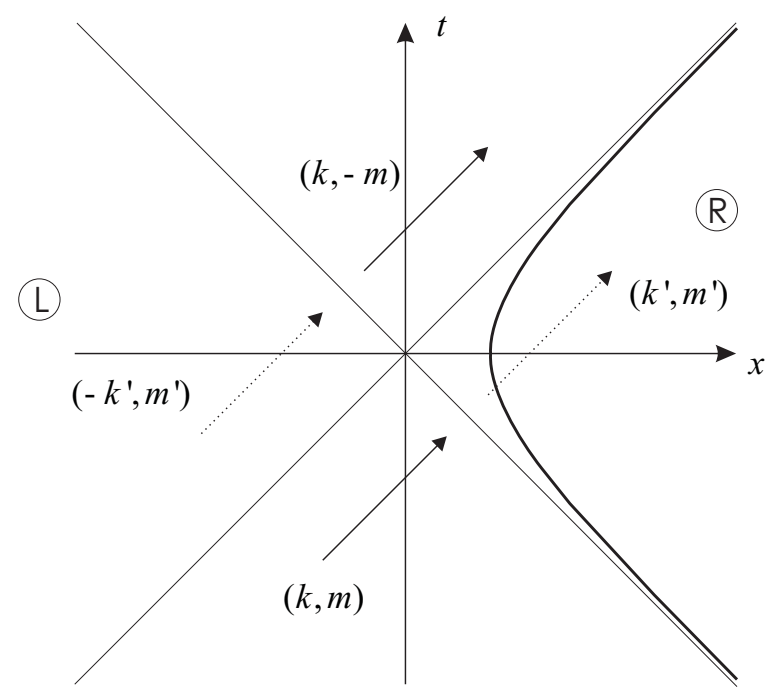

FIG. 1: Hyperbolic trajectory of a uniformly accelerated observer is shown in a thick line. With the standard choice of reference frames Alice crosses the $x$-axis at $x=1 / a$. Her past and future horizons define the right and left Rindler wedges. Solid arrows represent Minkowski particles with the trajectory parameters $(k, \pm m)$, while the dotted arrows represent the same particles as described by Alice. The mode in our example corresponds to $(k,-m)$ arrow.

transformation is to define new surfaces of simultaneity with respect to the Lorentz-transformed observer. These surfaces may intersect the event horizon differently from those of the original observer. However, the area theorem of Hawking [9] guaranties that all these intersections lead to the same horizon area. The semiclassical black hole entropy is given by Eq. (3) and the leading corrections are functions of the area only 22]. Hence the invariance of the horizon area keeps the entropy invariant. Since we are dealing here with a curved spacetime, there is no contradiction with the proposition that has been proven above.

The relations between entropy, energy and temperature are important in at least two contexts. The derivation [13] of the Einstein equations from the holographic principle and thermodynamics requires an independent expression for the temperature. It is taken to be the Unruh temperature of an accelerated observer in vacuum, while the matter is located beyond the observer's horizon. It is argued 16, 23 that a necessary condition for the generalized second law $[8,9]$ is that there are no highly entropic objects, i.e.,

$$
S \leq E / T,
$$

should hold, where $T$ is the black hole temperature and $E$ and $S$ are object's energy and entropy. This is to say that object's free energy is non-negative, $F=E-T S \geq 0$.

Consider a free real massless scalar field in $1+1$ Minkowski spacetime from the point of view of a uniformly accelerated observer (whom we conventionally name Alice). Minkowski particles are present beyond 
Alice's horizon in the left Rindler wedge. We investigate the relationship between the renormalized entropy, the renormalized energy and the temperature as observed by Alice (see Fig. 1 for definitions). Thanks to the results of Audretsch and Müller 24] all the relevant quantities can be calculated explicitly. Localized particles are most conveniently described in the wave packet basis [11, 18]. The Minkowski wave packet is defined by the superposition of the positive energy plane wave solutions $f_{k}=\frac{1}{\sqrt{4 \pi \omega_{k}}} e^{-i\left(\omega_{k} t-k x\right)}$, where $\omega_{k}=|k|$, in a momentum interval $\epsilon$,

$$
f_{k m}(t, x)=\frac{1}{\sqrt{\epsilon}} \int_{k}^{k+\epsilon} d \tilde{k} e^{-i \tilde{k} m} f_{\tilde{k}}(t, x) .
$$

For each wave packet both $k / \epsilon$ and $m \epsilon / 2 \pi$ are fixed integers. These wave packets form a complete orthonormal basis, and the quantum field is written with $a_{k m}$ and $a_{k m}^{\dagger}$ operators. For a small value of $\epsilon$, the state $|k, m\rangle=$ $a_{k m}^{\dagger}|\Omega\rangle$ represents a particle with energy $\langle E\rangle \approx \omega_{k}$ whose spatial localization roughly corresponds to the position of the maximum of the wave packet $f_{k m}$. All the reasonable localization POVM's [5] give only a power-law decrease of the probability density to find a massless particle at some distance from the classical trajectory that is specified by $m$. In particular, it is impossible to confine any Minkowski state exactly to one of the Rindler wedges.

The right and left Rindler modes $f_{k^{\prime} m^{\prime}}^{R}, f_{k^{\prime} m^{\prime}}^{L}$ are defined similarly. Fig. 1 represents a correspondence between the energy-momentum vector of a Minkowski par- ticle, $k^{\mu}=\left(\omega_{k}, k\right)$ in Minkowski and Rindler reference frames. For $k>0, m>0$ the equivalent description is given by

$$
\left(k^{\prime}, m^{\prime}\right) \Leftrightarrow(k, m)=\left(e^{-a m^{\prime}} k^{\prime}, e^{a m^{\prime}} / a\right),
$$

where $a$ is Alice's proper acceleration. The Bogoliubov coefficients are non-zero essentially only between the equivalent modes.

The Minkowski vacuum then takes the form

$$
|\Omega\rangle=\prod_{\tilde{k}^{\prime}, \tilde{m}^{\prime}} \frac{1}{\left|\alpha_{\tilde{k}^{\prime}}\right|} \sum_{q=0}^{\infty}\left(\frac{\beta_{\tilde{k}^{\prime}}^{*}}{\alpha_{\tilde{k}^{\prime}}^{*}}\right)^{q}\left|q_{-\tilde{k}^{\prime} \tilde{m}^{\prime}}\right\rangle_{L} \otimes\left|q_{\tilde{k}^{\prime} \tilde{m}^{\prime}}\right\rangle_{R}
$$

where $\left|q_{-\tilde{k}^{\prime} \tilde{m}^{\prime}}\right\rangle_{L}$ is a state with $q$ particles in the mode $-\tilde{k}^{\prime} \tilde{m}^{\prime}$ in the left Rindler wedge, etc. The coefficients $\alpha_{\tilde{k}^{\prime}}$, $\beta_{\tilde{k}^{\prime}}$ are given in 24]. Accordingly, the reduced density matrices in both wedges are

$$
\rho_{\Omega}^{L, R}=\prod_{\tilde{k}^{\prime}, \tilde{m}^{\prime}} \frac{1}{Z_{\tilde{k}^{\prime}}} \sum_{q=0}^{\infty} e^{-\omega_{\tilde{k}^{\prime}} \beta q}\left|q_{\mp \tilde{k}^{\prime} \tilde{m}^{\prime}}\right\rangle\left\langle q_{\mp \tilde{k}^{\prime} \tilde{m}^{\prime}}\right|
$$

where $Z_{\tilde{k}^{\prime}}=\left(1-\exp \left(-\omega_{\tilde{k}^{\prime}} \beta\right)\right)^{-1}$, and the parameter $\beta$ defined by the proper acceleration, $\beta=2 \pi / a$. This is a thermal distribution with $T_{U}=1 / \beta$.

A state $\left|n_{k m}\right\rangle$ with $n$ Minkowski particles that (essentially) pass through the left wedge has a more complicated form [24]. After some approximations, its reduced density matrix in the right wedge is

$$
\rho_{(n ; k m)}^{R}=\prod_{\tilde{k}^{\prime} \neq k^{\prime}, \tilde{m}^{\prime} \neq m^{\prime}} \frac{1}{Z_{\tilde{k}^{\prime}}} \sum_{q=0}^{\infty} e^{-\omega_{\tilde{k}^{\prime}} \beta q}\left|q_{\tilde{k}^{\prime} \tilde{m}^{\prime}}\right\rangle\left\langle q_{\tilde{k}^{\prime} \tilde{m}^{\prime}}\left|\otimes \frac{1}{Z_{k^{\prime}}^{n+1}} \sum_{r=0}^{\infty} \frac{(n+r) !}{n ! r !} e^{-\omega_{k^{\prime}} \beta r}\right| r_{k^{\prime} m^{\prime}}\right\rangle\left\langle r_{k^{\prime} m^{\prime}}\right|,
$$

where the "mirror mode" $\left(k^{\prime}, m^{\prime}\right)$ is related to $(k,-m)$ by Eq. (9). The number of excited modes is infinite, so the entropy diverges. A finite renormalized entropy of $\rho^{R}$ is defined as follows 15, 16]. First the regularized entropies of $\rho_{\Omega}^{R}$ and $\rho^{R}$ are calculated by imposing a cutoff $l$. Then the difference $S\left(\rho^{R} ; l\right)-S\left(\rho_{\Omega} ; l\right)$ of the cutoff-dependent entropies is taken. Finally the cutoff is removed. The renormalized energy is calculated similarly, with the Hamiltonian taken with respect to the Rindler time [16, 17]. Both quantities are independent of the cut-off, but their non-zero values result from the modes influenced by Minkowski particles.

In the limit $\exp (\beta \omega) \gg n$ it is easy to get explicit expressions for $S$ and $E$. For simplicity we consider the case with only one exited Minkowski mode. In this limit
$\Gamma$

$$
S=(\omega \beta n+n-(n+1) \ln (n+1)) e^{-\beta \omega},
$$

and

$$
E=\omega n e^{-\beta \omega},
$$

where the energy $\omega_{k^{\prime}}$ of the equivalent Rindler mode $\left(k^{\prime}, m^{\prime}\right)$ is denoted by $\omega$.

The notion of temperature is unapplicable for systems far from equilibrium, so the thermodynamic description is valid only when $\rho^{R}$ is well approximated by $\exp (-H / T) / \operatorname{tr} \exp (-H / T)$. In the free field theories the modes are independent. There is no way in which the Unruh temperature of the Minkowski vacuum, $T_{U}=1 / \beta=a / 2 \pi$, is relevant for the modes like $\left(k^{\prime}, m^{\prime}\right)$. Hence the correct interpretation is to split the system 
into two non-interacting parts. All the modes except for $\left(k^{\prime}, m^{\prime}\right)$ are thermal with a usual Unruh temperature $T_{U}$ form one subsystem. The mode $\left(k^{\prime}, m^{\prime}\right)$ forms another. When $n \exp (-\beta \omega) \ll 1$, this mode behaves as a twolevel system and its density matrix is close to the thermal distribution with the temperature $T$ that is introduced according to the definition in Eq. (4). A variable parameter is $n$, a number of the Minkowski particles in a given mode. While fractional expected number of particles between $n$ and $n+1$ is accomplished by mixing states with these particle numbers, it is consistent to vary $n$ as a real parameter in the approximation of Eqs. (13). The temperature is

$$
\frac{1}{T}=\frac{d S / d n}{d E / d n}=\beta-\frac{\ln (n+1)}{\omega} .
$$

As expected, with no Minkowski particles present, the temperature reduces to the Unruh temperature $T_{U}=$ $1 / \beta$. It is easy to see that for the state $\rho_{(n ; k m)}^{R}$

$$
S-E / T=(n-\ln (n+1)) e^{-\beta \omega}>0,
$$

i.e., its free energy is negative. It is consistent with $F=$ $-E / 3<0$ for the "usual" black body radiation [1].

Like in the field-in-a-box example, the entropy (13) is not invariant under Lorentz transformations. Consider now another accelerated observer (Bob). At the Minkowski time $t=0$ Alice passes through $x=1 / a$ with zero velocity (see Fig. 1). Bob has the same constant proper acceleration and passes thought the same spacetime point, but with the velocity $v>0$. The Rindler wedges of Bob and Alice overlap, but are not identical. In construction of their reduced density matrices different parts of the Universe are excluded, so their descriptions are not compatible 25]. The easiest way to get Bob's description of the quantum state $\left|n_{k m}\right\rangle$ is first to pass to the Minkowski frame where Bob's trajectory is that of the standard accelerated observer (i.e., looks like Alice's trajectory on Fig. 1). Then an application of Bogoliubov transformations gives Bob's state. Since Minkowski vacuum is Lorentz-invariant, it looks to Alice the same way as to Bob. However, the particles look differently: in the new Minkowski frame their energy acquires a Doppler factor $\sqrt{(1-v) /(1+v)}$, and the trajectory parameter $m$ is increased to reflect the change in the frame's origin. Using Eq. (9) it is easy to show that depending on the relative magnitude of $a m$ and $v$ the Rindler energy of the relevant Bob's mode is either greater or smaller than that of the corresponding Alice's mode. A substitution to Eqs. (13) and (15) shows that $S_{B} \neq S_{A}, T_{B} \neq T_{A}$ as well.

We showed that in Minkowski spacetime entropy of a reduced density matrix is not necessarily invariant quantity, so the entanglement between different degrees of freedom and their effective numbers are observerdependent. Taking this non-invariance into account weakens the arguments against a spacelike entropy bound 12], since they are based on the invariance of entropy. Presence of the matter fields outside the horizon may either change Unruh temperature or make thermodynamic description altogether inapplicable. Corrections to the Unruh temperature may point to the quantum corrections to the Einstein equations. On the other hand, the black hole entropy is invariant. This invariance may help to pick up the correct explanation of the black hole entropy from a multitude of different approaches [5, [9].

This work was initiated by discussions with Florian Girelli and David Poulin. Helpful suggestions and critical comments of Jacob Bekenstein, Netanel Lindner, Rob Myers, Amos Ori, Terry Rudolph, Lee Smolin, Rafael Sorkin and Rowan Thompson are gratefully acknowledged.

[1] L. D. Landau and E. M. Lifshitz, Statistical Physics, Part I (Butterworth-Heinemann, Oxford, 1980).

[2] A. Wehrl, Rev. Mod. Phys. 50, 221 (1978).

[3] T. M. Cover and J. A. Thomas, Elements of Information Theory (Wiley-Interscience, New York, 1991).

[4] A. Peres, Quantum Theory: Concepts and Methods (Kluwer, Dordrecht, 1993); M. A. Nielsen and I. L. Chuang, Quantum Computation and Quantum Information (Cambridge University Press, New York, 2000).

[5] A. Peres and D. R. Terno, Rev. Mod. Phys. 76, 93 (2004).

[6] D. R. Terno, in Quantum Theory: Reconsideration of Foundations, edited by A. Khrennikov (Växjö University Press, 2002) p. 397; e-print quant-ph/0111144

[7] G. G. Emch and C. Liu, The Logic of Thermostatistical Physics (Springer, Berlin, 2002).

[8] R. M. Wald, Quantum Field Theory in Curved Spacetime and Black Hole Thermodynamics (University of Chicago Press, Chicago, 1994).

[9] V. P. Frolov and I. D. Novikov, Black Hole Physics (Kluwer, Dordrecht, 1998); R. M. Wald, Living Rev. Relat. 4, 6 (2001).

[10] J. D. Bekenstein, Lett. Nuovo Cim. 4, 737 (1972); J. D. Bekenstein, Phys. Rev. D 9, 3292 (1974).

[11] S. W. Hawking, Commun. Math. Phys. 43, 199 (1975).

[12] R. Busso, Rev. Mod. Phys. 74, 825 (2002).

[13] T. Jacobson, Phys. Rev. Lett. 75, 1260 (1995).

[14] R. Haag, Local Quantum Physics: Fields, Particles, Algebras (Springer, Berlin, 1993).

[15] C. Holzhey et al., Nucl. Phys. B424, 443 (1994); C. Callan and F. Wilczek, Phys. Lett. B333, 55 (1994).

[16] D. Marolf et al., e-print hep-th/0310022

[17] N. D. Birrell and P. C. W. Davies, Quantum Fields in Cureved Space (Cambridge University, Cambridge, 1982);

[18] S. Takagi, Prog. Theor. Phys. Suppl. 88, 1 (1986).

[19] L. R. Bombelli et al., Phys. Rev. D 34, 373 (1986); M. Srednicki, Phys. Rev. Lett. 71, 666 (1993).

[20] U. Yurtsever, Phys. Rev. Lett. 91, 041302 (2003).

[21] S. J. Summers and R. Werner, Phys. Lett. A 110, 257 (1985); R. Verch and R. F. Werner, e-print quant-ph/0403089

[22] G. Gour and A. J. M. Medved, Class. Quantum Grav. 20, 3307 (2003). 
[23] D. Marolf and R. Sorkin, Phys. Rev. D 69, 024014 (2004).

[24] J. Audretsch and R. Müller, Phys. Rev. D 49, 4056

[25] A. Peres and D. R. Terno, J. Mod. Opt. 49, 1255 (2002). (1994). 